\title{
High-resolution neuromuscular ultrasound-based diagnosis of carpal tunnel syndrome in a sample of Egyptian population
}

\author{
Ahmed ElSadek, Mai Fathy * (10 and Amr AbdEIMoneim
}

\begin{abstract}
Background: Carpal tunnel syndrome is the commonest entrapment neuropathy. The cut-off point of crosssectional area to diagnose carpal tunnel syndrome may vary among populations. The objective of this study was to determine the best cut-off point of median nerve cross-sectional area to diagnose carpal tunnel syndrome among Egyptian population and to compare the difference of median nerve cross-sectional area in diabetic patients versus healthy individuals.
\end{abstract}

Results: Mean cross-sectional area was $7.78 \pm 1.260 \mathrm{~mm}^{2}$ among healthy individuals and $8 \pm 1.018 \mathrm{~mm}^{2}$ among diabetics. Cut-off cross-sectional area of median nerve above which compression is anticipated is $9.5 \mathrm{~mm}^{2}$.

Conclusion: Ultrasound can be used to diagnose carpal tunnel syndrome by measuring the median nerve crosssectional area.

Keywords: Carpal tunnel syndrome, Ultrasonography, Median nerve diameter

\section{Background}

Carpal tunnel syndrome (CTS) is the most common entrapment neuropathy. It accounts for $90 \%$ of referrals to neurophysiological studies, $1: 4 \%$ of the general population suffer from CTS [1]. Neurophysiological studies lack the anatomical description of the median nerve in CTS [2]. Accordingly, ultrasound (US) imaging of the median nerve offers the visualization of the median nerve at the carpal tunnel [3]. Moreover, recent studies recommend that US examination of the median nerve to become the standard to diagnose CTS [4]. The median nerve diameter at the pisiform bone was approved to be the reference to diagnose CTS [2].

\footnotetext{
*Correspondence: maifathy23@gmail.com
}

Neurology Dep., Faculty of Medicine, Ain Shams University, Cairo, Egypt

\section{Methods}

This is an observational cross-sectional study. This study included 194 wrists recruited from the outpatient clinics of Ain Shams University hospitals including diabetic patients not complaining from symptoms of CTS, patients presenting with symptoms of CTS and healthy controls. Participants were included if they were more than 18 years old. Participants presenting with symptoms of CTS were excluded if they had history of diabetes mellitus, thyroid disorder, renal or hepatic disorders, rheumatoid arthritis, gouty arthritis, chemotherapy intake, direct trauma to upper limb, symptoms suggestive of peripheral neuropathy or current pregnancy. All participants were divided according to clinical assessment by Arabic version of Boston carpal tunnel questionnaire (BCTQ) [7] to normal BCTQ $(\leq 19)$ and abnormal $(>19)$. Subjects with normal BCTQ were divided into diabetics and non-diabetics. Median nerve area was measured using ultrasound (Esaote, MyLab Five, Italy). Linear 5-12 $\mathrm{MHz}$ probe was placed on distal wrist between pisiform bone medially and scaphoid bone 
laterally to provide short-axis view of median nerve at its inlet to carpal tunnel. CSA of median nerve was measured from inner border of epineurium (Fig. 1). Subjects with abnormal BCTQ were subjected to neurophysiological studies and were divided into mild, moderate and severe [5]. Written informed consent was obtained from participants.

\section{Statistical analysis}

Descriptive data were represented as mean and standard deviation for continuous variables and as number and percentage for categorical ones. Comparisons were made between groups using $\mathrm{T}$ test. Receiver operating characteristics (ROC) with area under the curve (AUC) analyses were used to detect the sensitivity and specificity of cut-off value of median nerve diameter measured by US to determine its abnormality. The analysis was done on SPSS ver. 25(IBM SPSS, NY, USA, 2017). $p<0.05$ was considered significant.

\section{Results}

The study included 194 wrists, including 53 males (27.3\%) and $141(72.7 \%)$ females. The mean age was $41.63 \pm 11.28$. Among the participants, 128 (66\%) had normal BCTQ and 66 (34\%) had abnormal BCTQ.

Regarding subjects with normal BCTQ, 32(25\%) were males and 96 (75\%) were females with mean age of $40.08 \pm 11.502$. Among that group, 28(21.9\%) were diabetics. The mean age among non-diabetics with normal BCTQ was $38.88 \pm 11.148$ versus $44.36 \pm 11.929$ in diabetics. The mean median nerve CSA among nondiabetics with normal BCTQ was $7.78 \pm 1.260 \mathrm{~mm}^{2}$ versus $8 \pm 1.018 \mathrm{~mm}^{2}$ among diabetics with normal BCTQ. Comparison between the 2 groups was significant regarding age being higher among diabetics $(p=0.025)$, while there was no statistical significance regarding CSA between both groups ( $p=00.343$ ).

Regarding subjects with abnormal BCTQ, 21(31.8\%) were males and $45(68.2 \%)$ were females. The mean age was $44.65 \pm 10.247$. The mean BCTQ was $32.50 \pm 11.526$. The mean CSA was $15.58 \pm 3.053 \mathrm{~mm}^{2}$ (Table 1). Grading of CTS according to nerve conduction studies showed 41(62.1\%) mild CTS, 22(33.3\%) moderate CTS

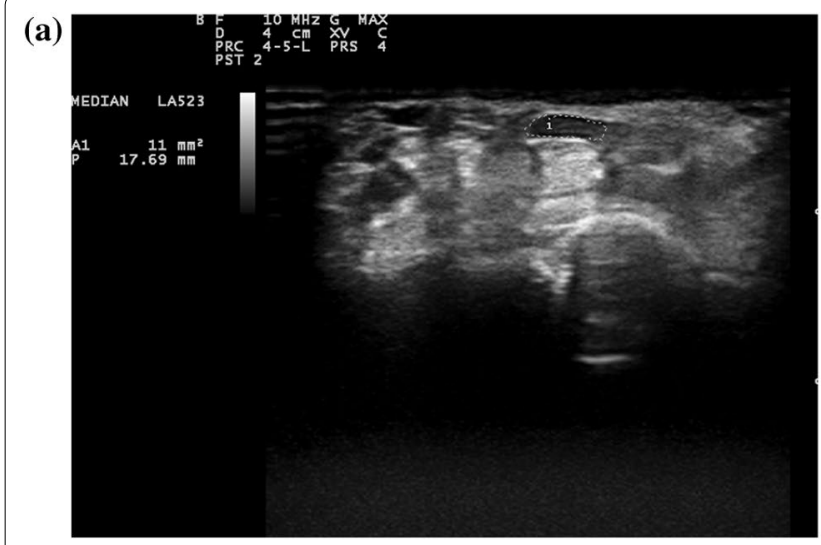

(b)

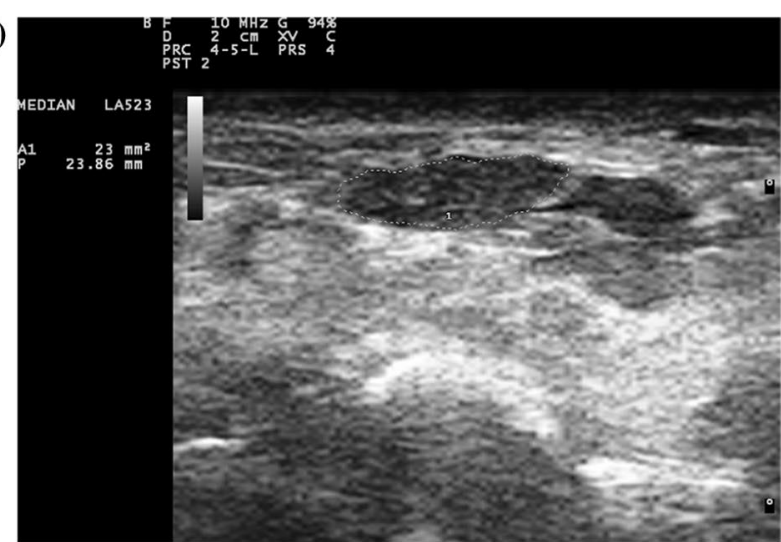

(c)

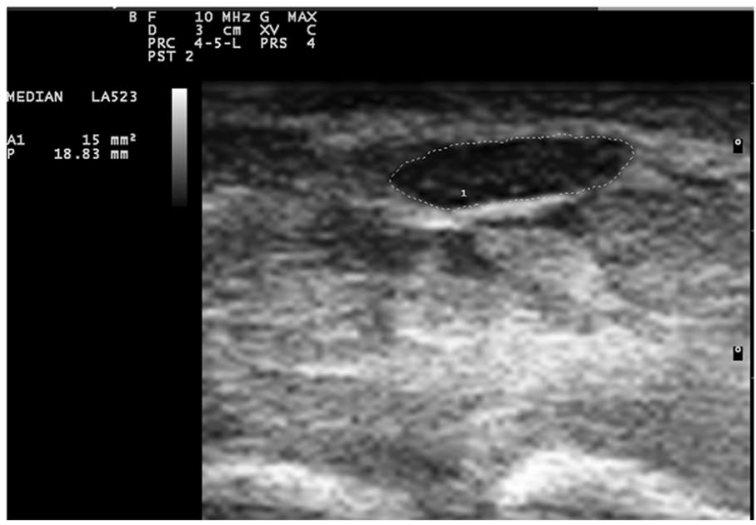

(d)

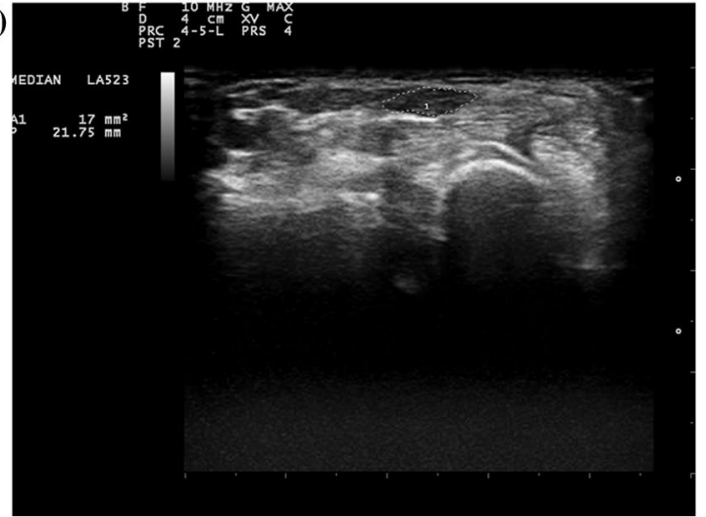

Fig. 1 Neurosonography figures showing anatomical and cross-sectional area measurements. a Right median nerve, BCTQ 27. b Left median nerve, BCTQ 21. c Right median nerve, BCTQ 38. d Right median nerve, BCTQ 20 
Table 1 Descriptive of subjects with abnormal BCTQ and neurophysiological studies (No.=66)

\begin{tabular}{|c|c|c|c|c|c|c|}
\hline & Mean & Median & SD & Range & Minimum & Maximum \\
\hline AGE & 44.65 & 45.00 & 10.247 & 41 & 24 & 65 \\
\hline CSA & 15.86 & 15.00 & 3.053 & 13 & 10 & 23 \\
\hline BCTQ & 32.50 & 29.00 & 11.526 & 48 & 20 & 68 \\
\hline Sensory latency & 4.76 & 4.60 & 0.88 & 4.80 & 3.60 & 8.40 \\
\hline Motor latency & 4.12 & 4.20 & 0.82 & 3.400 & 2.40 & 5.80 \\
\hline Motor amplitude & 9.68 & 9.15 & 4.43 & 20.50 & 0.50 & 21 \\
\hline Conduction velocity & 52.583 & 50.500 & 8.6893 & 42.0 & 35.0 & 77.0 \\
\hline
\end{tabular}

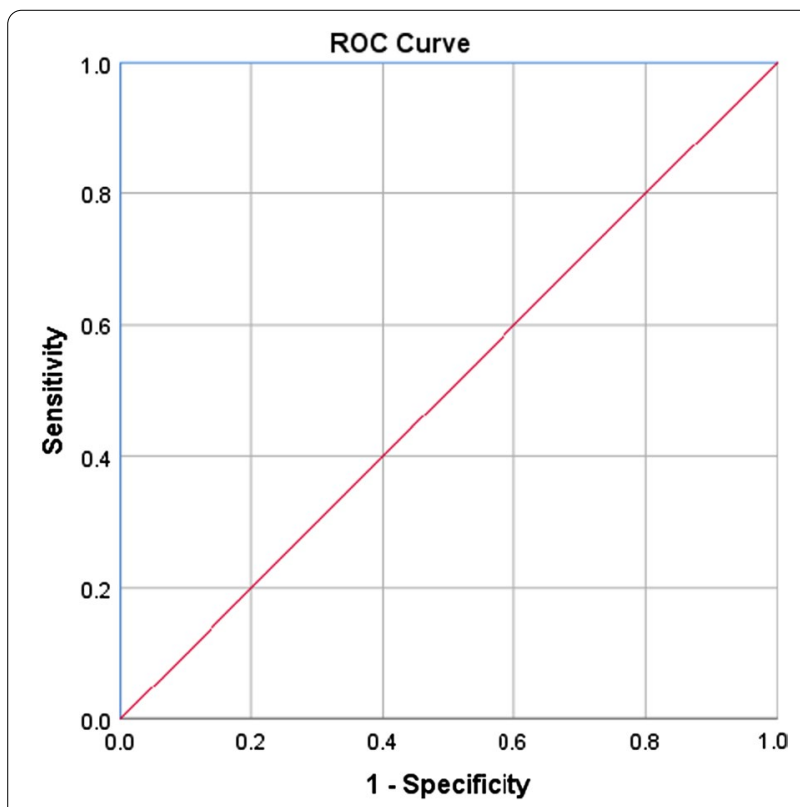

Fig. 2 ROC curve showing specificity and sensitivity of median nerve CSA

and 3(4.5\%) severe CTS. Patients with mild CTS had mean CSA of $15.59 \pm 2.924 \mathrm{~mm}^{2}$ and mean BCTQ of $31.83 \pm 11.129$, while patients with moderate CTS had mean CSA of $16.32 \pm 3.242 \mathrm{~mm}^{2}$ and mean BCTQ of $33.95 \pm 12.934$. ROC with AUC analysis was used to test the specificity and sensitivity of US diameter of median nerve to predict its abnormality. Area under the curve value was 1 indicating both strong test specificity and sensitivity in predicting compression with a p-value of $<0.0001$ (Fig. 2). Cut-off US CSA of median nerve above which compression is anticipated is $9.5 \mathrm{~mm}^{2}$ (at which the test sensitivity and specificity are $100 \%$ ).

\section{Discussion}

The utilizations of ultrasound to assess CTS through measuring median nerve CSA is a convenient non-invasive method. Few but conclusive studies stated a cut-off value to diagnose and grade the severity of CTS by CSA [6].

This study found a cut-off point of US CSA of median nerve above which compression is anticipated to be $9.5 \mathrm{~mm}^{2}$. Another Egyptian study by Sonbol and colleagues found that a CSA cut-off value of $10 \mathrm{~mm}^{2}$ provided a diagnostic sensitivity of $93.3 \%$ and specificity of 98.3\% [7]. Sarraf and colleagues found that the best cutoff point for the median nerve CSA $10.5 \mathrm{~mm}^{2}$ with $80 \%$ and $76 \%$ sensitivity and specificity, respectively. They stated that median nerve CSA at the wrist is helpful as a diagnostic tool for CTS [8]. Ziswiler and colleagues found that a cut-off point of $10 \mathrm{~mm}^{2}$ showed $82 \%$ and $87 \%$ sensitivity and specificity, respectively [9]. In Nakamichi and Tachibana study, the median nerve CSA cut-off point value of $12 \mathrm{~mm}^{2}$ showed $67 \%$ sensitivity and $97 \%$ specificity [10]. A study by Ghasemi and colleagues showed that participants with CSA $>10$ had CTS with $100 \%$ specificity and $83.12 \%$ sensitivity [11]. This shows that the cut-off point for diagnosing CTS may vary among different populations and that it is of utmost importance than each center must have their own reference. This study did not show statistical significance regarding CSA in different severities of CTS, In accordance with our findings, Mohammadi et al. studied the CSA of median nerve in severity grading of CTS and found that the difference in CSA of the median nerve among different severities of CTS was not statistically significant at either the tunnel inlet or outlet [12]. Also Sonbol and colleagues found no significant differences in CSA among patients with mild, moderate and severe CTS [7]. The prevalence of CTS is higher among diabetic patients specially those with diabetic polyneuropathy [13]. CSA was rarely found significantly higher in diabetic patients with CTS than in non-diabetic patients with CTS [14]. A study by Demino and colleagues, stated that cross-sectional area of the median nerve was similar for patients with and without DM [15]. However, no studies compared the median nerve CSA between diabetics and non-diabetics without CTS. Our study found no statistically significant 
difference between diabetics and non-diabetics regarding the median nerve CSA.

\section{Conclusion}

Measuring the median nerve cross-sectional area via ultrasound can be used as a convenient non-invasive method for the diagnosis of carpal tunnel syndrome with a cut-off value of 9.5. However, it may not be as useful regarding assessment of severity.

\section{Abbreviations}

BCTQ: Boston carpal tunnel questionnaire; CSA: Cross-sectional area; CTS: Carpal tunnel syndrome; US: Ultrasound.

\section{Acknowledgements}

Not applicable.

\section{Authors' contributions}

AS: conception of the work, manuscript revision and performing the ultrasound. MF: designed and conceptualized the study, drafting the manuscript, performing the ultrasound. AA: conception of the work, data collection, drafting the manuscript. All authors have agreed to conditions noted on the Authorship Agreement Form and have read and approved the final version submitted. The content of the manuscript has not been published, or submitted for publication elsewhere. All authors read and approved the final manuscript.

\section{Funding}

No funds were received to fulfill this work.

\section{Availability of data and materials}

The corresponding author takes full responsibility for the data, has full access to all of the data; and has the right to publish any and all data separate and apart from any sponsor.

\section{Declarations}

\section{Ethics approval and consent to participate}

All procedures performed in the study were in accordance with the ethical standards of the faculty of medicine, Ain Shams university research and ethical committee and with the 1964 Helsinki declaration and its later amendments or comparable ethical standards. We obtained approval from research ethics committee no. FWA 000017585. On 20/12/2020. Written informed consent was obtained from participants for participation.

\section{Consent for publication}

Not applicable.

\section{Competing interests}

None of the authors has any conflict of interest.
Received: 18 May 2021 Accepted: 1 October 2021

Published online: 16 October 2021

\section{References}

1. Yunoki M, Kanda T, Suzuki K, Uneda A, Hirashita K, Yoshino K. Importance of recognizing carpal tunnel syndrome for neurosurgeons: a review. Neurol Med Chir (Tokyo). 2017;57(4):172-83.

2. El-Bahnasawy AS, Senna MK, Okasha A, Ghariba O. Diagnostic utility of median nerve CSA to ulnar nerve CSA ratio in the diagnosis of mild idiopathic carpal tunnel syndrome. Egypt Rheumatol Rehabil. 2020;47:34.

3. El Badry A, El Sherif M, Yoshimine T. Can sonography replace electromyography and nerve conduction velocity in carpal tunnel syndrome? INDJ. 2016:6:1-10.

4. Yoshii Y, Zhao C, Amadio PC. Recent advances in ultrasound diagnosis of carpal tunnel syndrome. Diagnostics (Basel). 2020;10(8):596.

5. El-Habashy HR, El-Hadidy RA, Ahmed SM, El Sayed BB, Ahmed AS. Correlation between median nerve conduction studies and ultrasonography in cases of carpal tunnel syndrome. Egypt J Neurol Psychiatry Neurosurg. 2016;53:206-10.

6. Kang S, Kwon HK, Kim KH, Yun HS. Ultrasonography of median nerve and electrophysiologic severity in carpal tunnel syndrome. Ann Rehabil Med. 2012;36(1):72-9.

7. Sonbol M, Ibrahim W, Ghunaimi M. Role of ultrasonography in the diagnosis of carpal tunnel syndrome. Al-Azhar Med J. 2017:46(4):765-79.

8. Sarraf P, Malek M, Ghajarzadeh M, Miri S, Parhizgar E, Emami-Razavi SZ. The best cutoff point for median nerve cross sectional area at the level of carpal tunnel inlet. Acta Med Iran. 2014;52(8):613-8.

9. Ziswiler HR, Reichenbach S, Vogelin E, Bachmann LM, Villiger PM, Juni P. Diagnostic value of sonography in patients with suspected carpal tunnel syndrome: a prospective study. Arthritis Rheum. 2005;52(1):304-11.

10. Nakamichi K, Tachibana S. Ultrasonographic measurement of median nerve cross-sectional area in idiopathic carpal tunnel syndrome: diagnostic accuracy. Muscle Nerve. 2002;26(6):798-803.

11. Ghasemi M, Masoumi S, Ansari B, Fereidan-Esfahani M, Mousavi SM. Determination of cut-off point of cross-sectional area of median nerve at the wrist for diagnosing carpal tunnel syndrome. Iran J Neurol. 2017;16(4):164-7

12. Mohammadi A, Afshar A, Etemadi A, Masoudi S, Baghizadeh A. Diagnostic value of cross-sectional area of median nerve in grading severity of carpal tunnel syndrome. Arch Iran Med. 2010;13(6):516-21.

13. Bril V, Perkins B. Carpal tunnel syndrome in patients with diabetic polyneuropathy. J Peripher Nerv Syst. 2001:5(3):180-180.

14. Kotb MA, Bedewi MA, Aldossary NM, Mahmoud G, Naguib MF. Sonographic assessment of carpal tunnel syndrome in diabetic patients with and without polyneuropathy. Medicine (Baltimore). 2018;97(24):e11104.

15. Demino C, Fowler J. Diagnostic value of ultrasound in CTS in diabetic versus nondiabetic populations. J Hand Surg Glob Online. 2020;2(5):267-71.

\section{Publisher's Note}

Springer Nature remains neutral with regard to jurisdictional claims in published maps and institutional affiliations. 